Pacific Journal of Mathematics

STRONGLY SEMIPRIME RINGS 


\title{
STRONGLY SEMIPRIME RINGS
}

\author{
D. HANDELMAN
}

For a ring with 1, we show that every proper kernel functor generates a proper torsion radical if and only if the ring is a finite subdirect product of strongly prime (also called ATF) rings. This is equivalent to every essential right ideal containing a finite set whose right annihilator is zero. We use this characterization to quickly prove a number of properties of rings satisfying this condition, and apply the results to the problem: when is every kernel functor a torsion radical.

$R$ is an associative ring with 1 , and all modules are right modules. We use the notation $K<R$ to indicate $K$ is a (two-sided) ideal of $R$; if $A$ is a subset of $R$, then $A^{r}, A^{1}$ denote, respectively, the right and left annihilators of $A$.

For a nonzero element $r$ of $R$, a finite set $\left\{r_{i}\right\}$ in $R$ is a (right) insulator for $r$ if $\left\{r r_{i}\right\}^{r}=(0)$. A right ideal is insulated (in Beachy [1], 'cofaithful') if it contains a finite set whose right annihilator is zero, and the finite subset is also called an insulator. A ring is (right) strongly prime (SP) (Handelman and Lawrence [5]; also known as ATF in Rubin [10] and Viola-Prioli [12]) if every nonzero element has an insulator; equivalently every nonzero ideal is insulated. Details may be found in $[5,6,10,12]$.

A kernel functor (or left exact preradical) $U$, is a left exact subfunctor of the identity on $\operatorname{Mod}_{R}$, and to $U$ there corresponds a topologizing filter $\mathscr{E}$ : a nonempty filter of right ideals closed under finite intersection, and if $A \in \mathscr{E}$, then $r^{-1} A=\{s \in R \mid r s \in A\}$ belongs to $\mathscr{E}$. Details may be found in Goldman [2], Goodearl, Handelman and Lawence [6], and Viola-Prioli [12]. An ideal $K$ is a pretorsion ideal if there exists a kernel functor $U$ such that $U(R)=K$.

Torsion radicals, torsion ideals, torsion-free and torsion theories are discussed in Lambek [8]. Given a kernel functor $U$, the collection $\left\{M \in \operatorname{Mod}_{R} \mid U(M)=(0)\right\}$ is closed under isomorphic images, submodules, essential extensions, and direct products, hence is a torsionfree theory. By Proposition 0.3 of [8], there exists a unique torsion radical $T_{U}$ such that $T_{U}(M)=(0)$ if and only if $U(M)=(0)$ (equivalently $U(M)$ is an essential submodule of $T_{U}(M)$ for all modules $M$ ). A kernel functor is proper if $U(R) \neq R$.

Other underfined terms may be found in Lambek [7], except that 'essential ideal' denotes a two-sided ideal that is essential as a right ideal. 
A ring is (right) strongly semiprime (SSP) if every essential ideal is insulated. As semiprime is equivalent to every essential ideal being faithful, we see that an SSP ring is semiprime. In a prime ring, every nonzero ideal is essential, so a prime SSP ring is SP. We show that SSP rings are equivalent to the ETF rings of Rubin [11], and the semiprime rings satisfying the condition studied by Beachy and Blair [1]. More importantly, we show that the study of SSP rings reduces to that of SP rings, as SSP rings are finite subdirect products of SP rings.

THEOREM 1. For a ring $R$, the following are equivalent:

1. $R$ is a finite subdirect product of strongly prime rings

2. every essential right ideal is insulated

3. every essential ideal is insulated (SSP)

4. every proper pretorsion ideal is not essential

5. every proper kernel functor generates a proper torsion radical

6. $R$ is semiprime and every faithful right ideal is insulated.

Rings satisfying 4 were introduced, as 'ETF' rings, in Rubin [11]. Condition 5 states that if $U$ is a proper kernel functor, then $T_{U}(R) \neq R$. The equivalence of 4 with 5 was shown in Rubin's paper.

We prove Theorem 1 by showing $2 \Leftrightarrow 3 \Leftrightarrow 4 ; 1 \Rightarrow 3 ; 3 \Rightarrow 1 ; 5 \Leftrightarrow 4$; $3 \Leftrightarrow 6$. The implication $3 \Rightarrow 1$ is by far the most difficult.

Lemma 2. If $K$ is a proper pretorsion ideal, then for all finite subsets $\left\{k_{i}\right\} \subset K,\left\{k_{i}\right\}^{r} \neq(0)$.

Proof. Let $\mathscr{E}$ be the topologizing filter corresponding to $U$, where $U(R)=K$. Then $k_{i}^{r} \in \mathscr{E}$, so $\cap_{i} k_{i}^{r}=\left\{k_{i}\right\}^{r} \in \mathscr{E}$. If $\left\{k_{i}\right\}^{r}=(0),(0) \in \mathscr{E}$, so $U(R)=R$, i.e. $K$ is not proper.

LEMMA 3. A right ideal $J$ is contained in a proper pretorsion ideal if and only if $J$ is not insulated.

Proof. Define $\mathscr{C}_{J}=\left\{I<_{r} R \mid\right.$ there exists $\left\{j_{i}\right\}_{i=1}^{n} \subset J$, with $\left\{j_{i}\right\}^{r} \subset$ $I\}$. Then $\mathscr{E}_{J}$ is a topologizing filter. If $J$ is not insulated, then $(0) \notin \mathscr{E}_{J}$, so if $U_{J}$ is the corresponding kernel functor, $R J \subset U_{J}(R) \neq R$. The converse follows immediately from Lemma 2 .

PROPOSITION 4. For a ring $R$, the following are equivalent:

2. Every essential right ideal is insulated

3. every essential ideal is insulated

4. every proper pretorsion ideal is not essential. 
Proof. $2 \Rightarrow 3$ is trivial, and $3 \Rightarrow 4$ follows from the preceding lemmas. $4 \Rightarrow 2$ : If $E$ is an essential right ideal that is not insulated, then by Lemma 3, RE is an essential ideal that is contained in a proper pretorsion ideal.

PROPOSITION 5. If $R$ is a subdirect product of finitely many strongly prime rings, then $R$ is $S S P(1 \Rightarrow 3)$.

Proof. By assumption, there exist $L_{i}<R, i=1, \cdots n$, such that $\cap_{i} L_{i}=(0)$, and if $r \notin L_{i}$, there exists a finite set $\left\{s_{i j}\right\}_{j} \subset R$, such that $\cap\left(r s_{i j}\right)^{-1} L_{i}=L_{i}$. Now $R$ is semiprime; set $K_{i}=L_{i}$; ; then $K_{i} \cap L_{i}=$ (0). Let $E$ be an essential ideal and choose nonzero $r_{i} \in K_{i} \cap E$; there exists $\left\{s_{i j}\right\}$, a finite subset of $R$ such that for each $i, \cap_{j}\left(r_{i} s_{i j}\right)^{-1} L_{i}=L_{i}$; thus, $\cap_{j}\left(r_{i} s_{i j}\right)^{r} \subset L_{i}$. Hence

$$
\left\{r_{i} s_{i j}\right\}_{i, j}^{r} \subset \bigcap_{i} L_{i}=(0)
$$

So $\left\{r_{i} s_{i j}\right\}$ insulates $E$.

To prove the implication $3 \Rightarrow 1$ is more intricate. $Z(M)$ denotes the singular submodule of $M$, and $Z(R)$ is the right singular ideal.

Proposition 6. If $R$ is strongly semiprime, then

(i) $Z(R)=(0)$

(ii) $R$ contains no infinite direct sums of two-sided ideals.

Proof. (i). The topologizing filter corresponding to the kernel $Z()$ is the collection of essential right ideals. Set

$$
\mathscr{E}=\left\{I<_{r} R \mid Z(R) \cap E \subset I, \quad \text { for some essential } E\right\}
$$

Then $\mathscr{E}$ is a topologizing filter, and if $Z(R) \neq(0),(0) \notin \mathscr{E}$. The corresponding kernel functor yields the pretorsion ideal $U(R)=Z(R)+Z(R)^{1}$ - an essential ideal.

(ii). If $L=\oplus K_{i}$, with $K_{i}<R$, then $K=\left(\oplus K_{i}\right) \oplus L^{1}$ (observe that as $R$ is semiprime, $L \cap L^{1}=(0)$ ) is essential, and if $K$ is insulated, obviously the direct sum must be finite.

We now follow a process similar to that of Levy [9] to show a semiprime satisfying (ii) above is an essential subdirect product of finitely many prime rings.

We note that if $R$ is semiprime and $K<R$, then $K^{r}=K^{1}$ so the 'annihilator of an ideal' is unambiguous; we also note $K \subset K^{11}$. 
LEMMA 7. If $R$ is semiprime and contains no infinite direct sums of ideals, then $R$ satisfies the ascending and descending chain conditions on annihilators of ideals.

Proof. Suppose $K_{j}<R, j=1,2, \cdots$ and $K_{j}^{1} \subset K_{j+1}^{1}$. There exists $x_{j}$ in $R$ such that $x_{j} K_{j+1}=(0)$ but $x_{j} K_{j} \neq(0)$; as $R x_{j} K_{j} \cap K_{j}^{1}=(0)$, we have $R x_{j} K_{j} \oplus K_{j}^{1} \subset K_{j+1}^{1}$. Thus $\oplus R x_{j} K_{j}$ is an infinite direct sum of ideals. As left annihilators (of ideals) are also right annihilators, the descending chain condition also holds.

LEMMA 8. If $(0) \neq K<R$ and $K^{1}$ is maximal among annihilators of ideals, then $R / K^{1}$ is a prime ring.

Proof. If $V, W<R$ and $V W \subset K^{1}$, then $V W K=(0)$. If $W K=$ (0), then $W \subset K^{1}$; if $W K \neq(0)$, then $K^{1} \subset(W K)^{1} \neq R$, so by the maximality of $K^{1}, K^{1}=(W K)^{1}$, hence $V \subset K^{1}$; i.e., $K^{1}$ is a prime ideal.

Proposition 9. If $R$ is semiprime and contains not infinite direct sums of two-sided ideals, then $R$ is a subdirect product of finitely many prime rings $R_{i}$ such that the inclusion $R \rightarrow \pi R_{i}$ is an essential extension as right and left $R$-modules.

Proof. By Lemma 7, there exists a maximal annihilator ideal, $K_{1}^{1}$. If $\left\{K_{1}^{1}\right\}_{i=1}^{n}$ are maximal annihilators of ideals and $\cap_{i=1}^{n} K_{i}^{1} \neq(0)$, then there exists a maximal annihilator ideal $K_{n+1}^{1}$ such that $\cap_{i=1}^{n+1} K_{i}^{1} \neq \cap_{i=1}^{n} K_{i}^{1}$ : Find a minimal annihilator ideal $K_{n+1}$ inside the annihilator ideal, $\cap \cap_{i=1}^{n} K_{i}^{1}$; then $K_{n+1} \not \subset K_{n+1}^{1}$, a maximal annihilator, so $\cap_{i=1}^{n} K_{i}^{1} \not \subset K_{n+1}^{1}$. As $\left\{\cap_{i=1}^{n} K_{i}^{1}\right\}$ is a strictly descending chain of annihilator ideals, there must exist an $m$ such that $\cap_{i=1}^{m} K_{i}^{1}=(0)$. Now we may suitably refine the collection $\left\{K_{i}^{1}\right\}$ and thus assume that for all $j \leqq m, \cap_{i \neq j} K_{i}^{1} \neq(0)$. Thus $R \rightarrow \pi_{i=1}^{n} R / K_{i}^{1}$ is a subdirect representation (irreducible in the sense of Levy), and as $K_{i}^{1}$ is a maximal annihilator ideal, by Lemma 8 , each $R / K_{i}^{1}$ is a prime ring.

Finally, we prove $R \rightarrow \pi R / K_{i}^{1}$ is a right and left essential extension. Set $N_{j}=\bigcap_{i \neq j} K_{i}^{1} ; N_{j}$ is a nonzero annihilator ideal, and $N_{j} K_{j}^{1} \subset N_{j} \cap K_{j}^{1}=(0)$, so $N_{j} \subset K_{j}^{11}$. As $K_{j}^{1}$ is a maximal annihilator ideal, so we must have $K_{j}^{11}=N_{j}$. Pick $r \notin K_{j}^{1}$. Then $r \cdot N_{j} \not \subset K_{j}^{1}$ (if $r \cdot N_{j} \subset K_{j}^{1}$, then $r \cdot N_{j}=(0)$, so $\left.r \in N_{j}^{1}=K_{j}^{111}=K_{j}^{1}\right)$, thus there exists $t \in N_{j}$. such that $r t \notin K_{j}^{1}$, proving the right essentiality.

LEMмA 10. If $S$ is an overring of $R$, and $S$ is an essential extension of $R$ as right $R$-modules, then if $\mathscr{E}$ is a proper topologizing filter on $S$, 


$$
\mathscr{E}_{R}=\{A \cap R \mid A \in \mathscr{E}\}
$$

is a proper topologizing filter on $R$. The proof is straightforward.

COROLLARY 11. If $Q$ is the maximal ring of right quotients of $R$, and $R \subset S \subset Q$, and $R$ is $S S P$, then $S$ is SSP.

Proof. We observe that if the right annihilator in $R$ of a subset of $R$ is (0), then its right annihilator in $S$ is also (0).

\section{Lemma 12. If $R=\pi R_{1}$ is SSP, then $R_{1}$ is SSP.}

Proof. Pick an essential ideal $E<R_{1}$. Then $E \oplus\left(\oplus_{1>1} R_{i}\right)$ is an essential ideal of $R$, therefore contains an insulator. Pick the $R_{1}$ components of this insulator-these lie in $E$ and satisfy $\cap$ $\left(s_{1}\right)^{-1}\left(\oplus_{i \geqq 2} R_{i}\right)=\oplus_{i \geqq 2} R_{j}$, so in $R_{i},\left\{s_{j}\right\}^{r}=(0)$. Hence $E$ has an insulator, in $R_{1}$.

THEOREM 13. If $R$ is SSP, then $R$ is a finite subdirect product of strongly prime rings. $(3 \Rightarrow 1)$.

Proof. By Propositions 6, 9, $R$ is an essential finite subdirect product of rings $R_{i}$; by Corollary $11, \pi R_{i}$ is SSP; by Lemma $12, R_{i}$ is prime SSP, thus SP.

Proposition 14. (Proposition 2 of Rubin [11].) Every proper pretorsion ideal is not essential is equivalent to every proper kernel functor generating a proper torsion radical.

Proof. $\Rightarrow$ If $U(R)=K$ is not essential, choose $a R \subset R$ such that $a R \cap K=(0)$; then $U(a R)=(0)$, so $T_{U}(a R)=(0)$, hence $T_{U}(R) \neq R$.

$\Leftarrow$ : Let $K=U(R)$ be a proper essential torsion ideal; as in the proof of Proposition 6(i), $Z+U$ is a proper kernel functor (more precisely, it generates a proper kernel functor). As $T_{U}(K)=K$ and $K$ is essential implies $T_{Z}(R / K)=R / K$, both $K$ and $R / K$ are torsion at $T_{Z+U}$. Since torsion theories are closed under (group) extensions and $0 \rightarrow K \rightarrow R \rightarrow R / K \rightarrow 0$ is exact, we must have $T_{Z+U}(R)=R$; so $Z+U$ does not generate a proper torsion radical.

THEOREM 15. $R$ is strongly semiprime if and only if $R$ is semiprime and every faithful right ideal is insulated. $(3 \Leftrightarrow 6)$

Proof. $\Rightarrow$. If $J$ is a faithful right ideal and not insulated, then niether is $R J$ insulated; however in a semiprime ring, a faithful ideal is essential, contradicting the SSP definition. 
$\Leftarrow$ If $E$ is essential and $E^{r} \neq(0)$, then $R$ is not semiprime; hence every essential is faithful and thus insulated.

This completes the proof of Theorem 1 .

Corollary 16. A regular SSP ring is a finite product of simple rings.

Proof. If $R$ is regular, each SP ring $R_{i}$ is regular, hence simple by e.g. Handelman and Lawrence [5; II. 3]; and a finite subdirect product of simple rings is a finite product of simple rings.

COROLlARY 17. If $R$ is strongly semiprime, its maximal ring of right quotients is a finite product of simple rings.

COROLlARY 18. If $R$ is strongly semiprime and every nonzero ideal contains a nonzero uniform ideal, then $R$ is Goldie.

COROllary 19. SSP is a Morita invariant.

COROLlaRY 20. If $R$ is strongly semiprime and $G$ is a cancellative ordered semigroup or group, then $R G$ is strongly semiprime.

Corollaries 19 and 20 follow from the corresponding results on SP rings and the subdirect representation. (e.g. Viola-Prioli [12]). In particular, if $S$ is a polynomial ring over $R$ in any number of variables, commuting or noncommuting, then $R$ is SSP if and only if $S$ is SSP.

Corollary 21. A commutative SSP ring is Goldie.

Corollary 22. A finite subdirect product of SSP rings is SSP.

COROLlaRY 23. If $R$ is semiprime and has minimal condition on right annihilators, then $R$ is strongly semiprime.

Proof. Clearly $R$ has no infinite direct sums of two-sided ideals; hence by Proposition $9, R$ is a finite subdirect product of prime rings, $R / K_{i}^{1}$. As we are factoring out annihilators, the factor rings inherit the minimal condition. But by Handelman and Lawrence [5; I.1], a prime ring with minimal condition on right annihilators is $S P$, so $R$ is a finite subdirect product of $S P$ rings.

COROllary 24. A semiprime Goldie ring is right and left strongly semiprime. 
For ETF rings, Rubin [11], proved the results corresponding to Proposition 6 and Corollaries 11, 17, 19, 21, and 24.

EXAMPLES. In view of Theorem 1, the study of SSP rings reduces to that of SP rings. All SP rings are SSP, and examples include simple rings, prime Goldie rings, free products of nontrivial algebras with 1, domains, etc. In Handelman and Lawrence [5] a right but not left SP ring is constructed, and examples are also given in Handelman [4]. These are necessarily right but not left SSP rings.

The dual condition to number 4 of Theorem 1, every nonzero pretorsion ideal is essential, is considered in Handelman [3]; nonsingular rings with this property are exactly the rings whose maximal ring of quotients is prime regular.

In Viola-Prioli [12], the condition that every kernel functor be a torsion radical is considered. By Theorem 1, rings with this property are SSP (Condition 5), and a prime ring with this property is SP, with every ideal idempotent. It is tempting to conjecture that rings satisfying Viola-Prioli's condition be noetherian $V$-rings (equivalently, a finite product of simple noetherian $V$-ring). However, if $R$ is commutative, we immediately obtain a result found in [12]:

COROLlaRY 25. If $R$ is commutative and every kernel functor is a torsion radical, then $R$ is a finite product of fields.

Proof. A commutative prime ring is a domain, and a domain with its ideals idempotent, in the commutative case, is a field. So $R$ is a finite subdirect product of fields, hence a finite product of fields.

It is perhaps worth noting that the second half of condition 6 of Theorem 1 (studied in Beachy [1]) can be reinterpreted in terms of torsion ideals. Analogous to Lambek's nomenclature [8] for torsion ideals, we call an ideal $L$ pretorsion of type $I$ if there exists $K<R$ such that $L=K^{1}$ (such an $L$ is always a pretorsion ideal).

BROPOSITION 26. For a ring $R$, the following are equivalent:

(i) every faithful right ideal is insulated

(ii) every proper pretorsion ideal is contained in a proper pretorsion ideal of type $I$.

Proof. (i) $\Rightarrow$ (ii). If $K$ is a proper pretorsion ideal, it cannot be insulated by Lemma 2 , so it is not faithful; hence $K \subset K^{r} \neq R$. (ii) $\Rightarrow$ (i): If $J$ is a right ideal that is not insulated, by Lemma $3, J$ is contained in a proper pretorsion ideal; by (ii) $J^{r} \neq(0)$, i.e., $J$ is not faithful. 
In a ring with minimum condition on right annihilators, every pretorsion ideal is of type I ([6]); hence any such ring satisfies the conditions of Proposition 25. Beachy and Blair [1] exhibit a left noetherian ring which does not satisfy these conditions on the left (but must on the right).

Added in Proof. (1). Using the result that for polynomial identity rings, if all ideals are idempotent then the ring is regular (ArmendarizFisher, 'Regular P.I.-rings', Proc. Amer. Math. Soc. July, 1973), it can easily be deduced from Theorem 1 that a PI ring all of whose kernel functors are torsion radicals is semisimple artinian.

(2). M. Fenrick (Communications in Algebra, Volume 2, No. 4 1974) has characterized noetherian rings all of whose kernel functors are idempotent.

\section{REFERENCES}

1. J. Beachy and W. D. Blair, Rings whose faithful left ideals are cofaithful, preprint.

2. O. Goldman, Rings and modules of quotients, J. Algebra, 13 (1969), 10-47.

3. D. Handelman, When is the maximal ring of quotients projective?, to appear, Proc. Amer. Math. Soc.

4. - Prime regular rings of quotients to appear, Comm. in Algebra.

5. D. Handelman and J. Lawrence, Strongly prime rings to appear, Trans. Amer. Math. Soc.

6. K. Goodearl, D. Handelman and J. Lawrence, Strongly Prime and Completely Torsion-Free Rings, Monograph, Carleton University Press (1974).

7. J. Lambek, Lectures on Rings and Modules, Blaisdell (1966) Waltham, Mass.

8. - Torsion Theories, Additive Semantics and Rings of Quotients, Springer-Verlag \# 177 (1971).

9. L. Levy, Unique direct sums of prime rings, Trans. Amer. Math. Soc., 106 (1963), 64-76.

10. R. Rubin, Absolutely Torsion-free rings, Pacific J. Math., 46 (1973), 503-14.

11. Essentially torsion-free rings, preprint.

12. J. Viola-Prioli, On absolutely torsion-free rings and kernel functors, Ph.D. thesis (June 1973), Rutgers University, New Brunswick, New Jersey.

Received May 17, 1974.

MCGILl UNIVERSITY 
D. E. Bennett, Strongly unicoherent continua ............................. 1

Walter R. Bloom, Sets of p-spectral synthesis ................................ 7

R. T. Bumby and D. E. Dobbs, Amitsur cohomology of quadratic extensions: Formulas and number-theoretic examples ................. 21

W. W. Comfort, Compactness-like properties for generalized weak topological sums

D. R. Dunninger and J. Locker, Monotone operators and nonlinear biharmonic boundary value problems ...

T. S. Erickson, W. S. Martindale, 3rd and J. M. Osborn, Prime nonassociative algebras

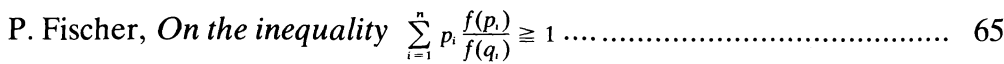

G. Fox and P. Morales, Compact subsets of a Tychonoff set ............... 75

R. Gilmer and J. F. Hoffmann, A characterization of Prüfer domains in terms of polynomials ......................................................... 81

L. C. Glaser, On tame Cantor sets in spheres having the same projection in each direction ......................................................... 87

Z. Goseki, On semigroups in which $X=X Y X=X Z X$ if and only if

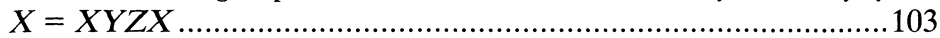

E. Grosswald, Rational valued series of exponentials and divisor

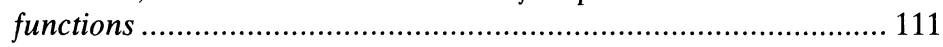

D. Handelman, Strongly semiprime rings ...................................... 115

J. N. Henry and D. C. Taylor, The $\bar{\beta}$ topology for $w^{*}$-algebras ............. 123

M. J. Hodel, Enumeration of weighted p-line arrays .......................... 141

S. K. Jain and S. Singh, Rings with quasiprojective left ideals .............. 169

S. Jeyaratnam, The diophantine equation $Y(Y+m)(Y+2 m) \times$

$$
(Y+3 m)=2 X(X+m)(X+2 m)(X+3 m) \ldots \ldots \ldots \ldots \ldots \ldots \ldots . . .183
$$

$\mathrm{R}$. Kane, On loop spaces without $p$ torsion .........................................189

Alvin J. Kay, Nonlinear integral equations and product integrals ..........203

A. S. Kechris, Countable ordinals and the analytic hierarchy, I ...........223

Ka-Sing Lau, A representation theorem for isometries of $C(X, E) \ldots \ldots . .229$

I. Madsen, On the action of the Dyer-Lashof algebra in $H_{*}(G)$..........235

R. C. Metzler, Positive linear functions, integration, and Choquet's theorem ........................................................................................ 277

A. Nobile, Some properties of the Nash blowing-up ............................297

G. E. Petersen and G. V. Welland, Plessner's theorem for Riesz conjugates 


\section{Pacific Journal of Mathematics}

\section{Vol. 60, No. $1 \quad$ September, 1975}

Donald Earl Bennett, Strongly unicoherent continua ................ 1

Walter Russell Bloom, Sets of p-spectral synthesis ................ 7

Richard Thomas Bumby and David Earl Dobbs, Amitsur cohomology of

quadratic extensions: formulas and number-theoretic examples .......

W. Wistar (William) Comfort, Compactness-like properties for generalized

weak topological sums ...................................

Dennis Robert Dunninger and John Stewart Locker, Monotone operators

and nonlinear biharmonic boundary value problems ..............

Theodore Erickson, Wallace Smith Martindale, III and J. Marshall Osborn,

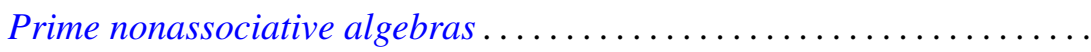

Pál Fischer, On the inequality $\sum_{i=0}^{n}\left[f\left(p_{i}\right) / f\left(q_{i}\right)\right] p_{i} \geq i \ldots \ldots \ldots \ldots \ldots$

Geoffrey Fox and Pedro Morales, Compact subsets of a Tychonoff set.......

Robert William Gilmer, Jr. and Joseph F. Hoffmann, A characterization of

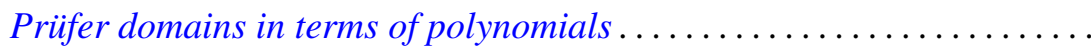

Leslie C. Glaser, On tame Cantor sets in spheres having the same projection

in each direction . ...................................

Zensiro Goseki, On semigroups in which $x=x y x=x z x$ if and only if

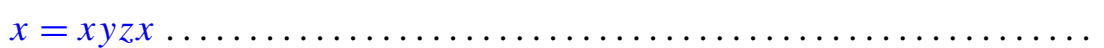

Emil Grosswald, Rational valued series of exponentials and divisor

functions.

David E. Handelman, Strongly semiprime rings

Jackson Neal Henry and Donald Curtis Taylor, The $\bar{\beta}$ topology for

$W^{*}$-algebras

Margaret Jones Hodel, Enumeration of weighted p-line arrays ...

Surender Kumar Jain and Surjeet Singh, Rings with quasi-projective left

ideals.

S. Jeyaratnam, The Diophantine equation

$$
Y(Y+m)(Y+2 m)(Y+3 m)=2 X(X+m)(X+2 m)(X+3 m) \ldots
$$

Richard Michael Kane, On loop spaces without $p$ torsion

Alvin John Kay, Nonlinear integral equations and product integrals ...

Alexander S. Kechris, Countable ordinals and the analytical hierarchy.

$I$.

Ka-Sing Lau, A representation theorem for isometries of $C(X, E)$

Ib Henning Madsen, On the action of the Dyer-Lashof algebra in $H_{*}(G)$

Richard C. Metzler, Positive linear functions, integration, and Choquet's

theorem.

Augusto Nobile, Some properties of the Nash blowing-up

Gerald E. Peterson and Grant Welland, Plessner's theorem for Riesz. 